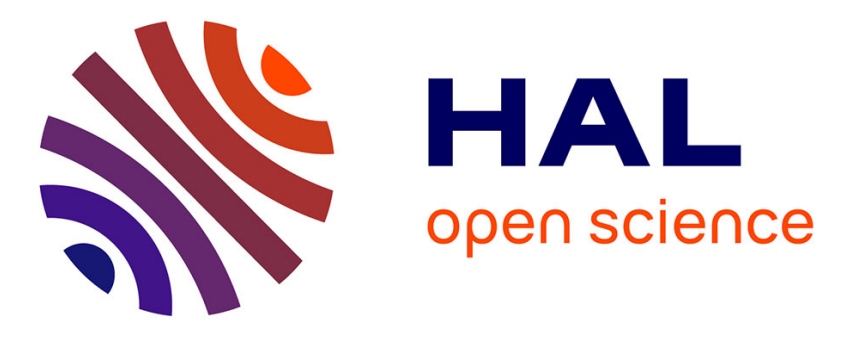

\title{
Turbo-FSK: A new uplink scheme for low power wide area networks
}

Yoann Roth, Jean-Baptiste Doré, Laurent Ros, Vincent Berg

\section{To cite this version:}

Yoann Roth, Jean-Baptiste Doré, Laurent Ros, Vincent Berg. Turbo-FSK: A new uplink scheme for low power wide area networks. SPAWC 2015 - 16th Signal Processing Advances in Wireless Communications, IEEE, Jun 2015, Stockholm, Sweden. 10.1109/SPAWC.2015.7227004 . hal-01289883

\section{HAL Id: hal-01289883 \\ https://hal.science/hal-01289883}

Submitted on 17 Mar 2016

HAL is a multi-disciplinary open access archive for the deposit and dissemination of scientific research documents, whether they are published or not. The documents may come from teaching and research institutions in France or abroad, or from public or private research centers.
L'archive ouverte pluridisciplinaire HAL, est destinée au dépôt et à la diffusion de documents scientifiques de niveau recherche, publiés ou non, émanant des établissements d'enseignement et de recherche français ou étrangers, des laboratoires publics ou privés. 


\title{
Turbo-FSK: a New Uplink Scheme for Low Power Wide Area Networks
}

\author{
Yoann ROTH ${ }^{1}$, Jean-Baptiste DoRÉ ${ }^{1}$, Laurent $\operatorname{Ros}^{2}$ and Vincent BERG ${ }^{1}$ \\ ${ }^{1}$ CEA, LETI, MINATEC Campus, F-38054 Grenoble, France. \\ ${ }^{2}$ Univ. Grenoble Alpes, GIPSA-Lab, F-38000 Grenoble, France \\ \{yoann.roth, jean-baptiste.dore, vincent.berg\}@cea.fr, laurent.ros@gipsa-lab.grenoble-inp.fr
}

\begin{abstract}
The Internet of Things aims to connect several billions of devices. Terminals are expected to be low cost, low power, and able to achieve successful communication at long range. While current Machine-to-Machine technologies tend to use spreading factors to meet the required specifications, we propose a more sophisticated use of redundant waveforms in a scheme called Turbo-FSK. This scheme involves FrequencyShift-Keying (FSK) modulation at the transmission, and a turbodecoder dedicated to the FSK waveforms at the receiver. Highly robust communication is achieved with a mere transmitter, as complexity is deported on the receiver side. Results are compared to common modulations using spreading factors, a significant gain in performance is achieved even with small packet sizes.
\end{abstract}

Keywords-Turbo code, FSK, Low rate, Low SNR, Internet of Things, IoT, Machine-to-Machine, M2M, Low Power Wide Area, LPWA, Shannon's limit, Channel Capacity

\section{INTRODUCTION}

Machine-to-Machine (M2M) communication is growing exponentially such that several billions of devices are expected to be connected to the Internet-of-Things (IoT) in the next decade [1], [2]. In this field, research on the design of a Low Power Wide Area (LPWA) network is required where challenges for terminals include low power consumption, low cost and fair sensitivity. The choice of the physical layer has a critical impact on these issues: it needs to be robust, efficient, and working at very low levels of sensitivity. It can be assumed that the numerous connected devices only transmit in a sporadic way, sending a few packets from time to time [2]: a low data rate communication per user is expected. This scenario is currently considered by the first generation of IoT commercial networks such as [3], [4], [5].

Reducing the level of sensitivity is challenging as the radio-frequency front end needs to be optimized, and the physical layer, the modulation and associated signal processing have to be carefully chosen. $M$-ary Orthogonal modulations appear to be a natural choice for low data rate and low power applications, as they offer a possible trade-off between energy and bandwidth. These modulations are power efficient and are known to reach the channel capacity for infinite sizes of alphabet $M$ (hence infinite size of signaling waveform duration) [6]. However, since an infinite size alphabet is an unrealistic option, a well known alternative is to increase energy efficiency by means of channel coding [7], notably using turbo processing [8].

The idea of combining orthogonal codes in a parallel concatenated scheme with turbo decoding was first proposed in [9], using Hadamard binary codes. A further study [10] observed strong error floors because of the poor minimal distance of the code. The authors proposed the addition of a convolutional code before the Hadamard mapping and achieved outstanding performance for large block sizes and large sizes of alphabet; limits of such a code have been studied in [11] and extensions have been proposed in [12]. On the other hand, Frequency-Shift-Keying (FSK) is an interesting choice of orthogonal modulation because of its constant envelope property, the simplicity of the modulator and its robustness to frequency-selective multi-path channels. Demodulation may simply be based on Fast-Fourier-Transform (FFT) as in an Orthogonal-Frequency-Division-Multiplexing receiver [13]. FSK has been exploited in a turbo coding scheme, BitInterleaved Coded Modulation with Iterative Decoding (BICMID) (see [14], [15]), where the steps of binary channel coding and $M$-ary FSK modulation are separated by an interleaver to ensure diversity at the receiving side. The receiver shares information of the decoded soft bits between the decoder and the orthogonal soft demodulator. Even though this scheme offers good performance, complexity is high because of the use of a 64-state convolutional encoder, and a large block size is used.

In this paper, we propose a new scheme called Turbo-FSK, and perform its analysis. Unlike [15], where a binary coding step was performed before FSK signaling, the new idea is to directly consider FSK signals as codewords. This scheme is an extension of the Turbo Hadamard scheme from [10] to the FSK modulation, replacing the binary Hadamard codes by the FSK waveforms. The transmitter uses then orthogonal FSK waveforms as codewords in a concatenated scheme, and the receiver is based on turbo decoding of these FSK codewords. To the best of our knowledge, FSK has never been presented before as potential orthogonal modulation for the scheme proposed in [10]. Neither decoder computations nor performance have been introduced. Also, the use of such a scheme as a physical layer for low data rate communications has never been explored. In our present study, after the presentation of the low complexity transmitter, the equations of the turbo decoder are derived for the FSK case. Performance of the Turbo-FSK is compared to other known modulations, including BICM-ID and spreading factors-based schemes, showing an interesting alternative. The use of Turbo-FSK scheme for LPWA is justified as the uplink M2M communication needs to be power efficient, low data rate and with fair sensitivity.

The paper is organized as follows. The motivations that led us to the use of such a scheme are presented in Section II. The system model is described in Section III and Section IV introduces the Turbo-FSK soft decoding computations. Simulation results are presented in Section V and Section VI concludes the paper. 


\section{MOTIVATIONS}

Because long range is linked to low sensitivity level and the terminal must be kept simple, a widely used technique to improve sensitivity of a primary technique is achieved by means of a spreading, or repetition factor, $\lambda$. At the reception in presence of Additive White Gaussian Noise (AWGN), the required Signal-to-Noise Ratio (SNR) using a spreading factor (denoted $\mathrm{SNR}_{\lambda}$ ) is decreased (i.e improved) as

$$
\mathrm{SNR}_{\lambda}^{(\mathrm{dB})}=\mathrm{SNR}_{1}^{(\mathrm{dB})}-10 \log _{10}(\lambda),
$$

where $\mathrm{SNR}_{1}$ is the required SNR of the primary technique. This is done at the cost of a data transmission rate reduction equal to the factor $\lambda$. However, the impact on the spreading factor is different for the $E_{b} / N_{0}$, the energy per bit to noise spectral density ratio (or SNR per bit), defined by

$$
\frac{E_{b}}{N_{0}}=\frac{\mathrm{SNR}}{\eta},
$$

where $\eta$ is the normalized spectral efficiency, in bit/s/Hz. Indeed, if a spreading factor is used, $\eta$ is divided by $\lambda$, with then $\eta_{\lambda}=\eta_{1} / \lambda$, where $\eta_{1}$ (resp. $\eta_{\lambda}$ ) is the spectral efficiency of the primary technique (resp. using the repetition $\lambda$ ). As a consequence, eq. (1) and (2) clearly demonstrate that the processing gain on the SNR with a spreading factor will be compensated in $E_{b} / N_{0}$ by the reduction of the rate. If the spreading factor scheme allows a decrease of the required SNR, it does not permit a decrease in terms of $E_{b} / N_{0}$.

The Shannon capacity [16], or Shannon's limit, is the maximal transmission rate with arbitrarily small bit-error probability, for a given SNR and a given bandwidth. It is straightforward that reaching this limit for a certain rate allows for a better energy efficiency of the system. The limit can be reformulated in terms of maximum spectral efficiency, or in terms of minimum $E_{b} / N_{0}$ as

$$
\begin{aligned}
\eta & \leq \log _{2}(1+\mathrm{SNR}) \\
\Leftrightarrow \frac{E_{b}}{N_{0}} & \geq \frac{2^{\eta}-1}{\eta},
\end{aligned}
$$

and is depicted Figure 1 along with the performance of several common modulations: Phase-Shift-Keying (PSK), $M$ ary orthogonal modulation, and Binary-PSK (BPSK) combined with a spreading factor (primary technique BPSK repeated by $\lambda$ ). Values are obtained by simulations of the considered modulations and denoting the required $E_{b} / N_{0}$ for a BitError-Rate (BER) of $10^{-5}$. The figure highlights the fact that the spreading factor only improves sensitivity, but does not enhance energy efficiency; for ultra-low rates, the gap with Shannon's limit reaches a constant value of $\simeq 11.1 \mathrm{~dB}$. On the contrary, for $M$-ary orthogonal modulations, increasing the alphabet size induces an improvement (i.e a reduction) in $E_{b} / N_{0}$ while the rate is decreased. However, very high values of $M$ (thus very small spectral efficiency) are required to approach the Shannon's limit. The proposed Turbo-FSK scheme combines both orthogonal modulation and turbo processing in the perspective to get closer to Shannon's limit with a reasonable size of alphabet.

\section{SYSTEM MODEL}

First let us introduce some notations. Bold lowercase letters denote vectors, e.g. $\boldsymbol{x}$, and bold uppercase letters matrices, e.g. $\boldsymbol{X}$. Exponents indicate the row number, indices the column.

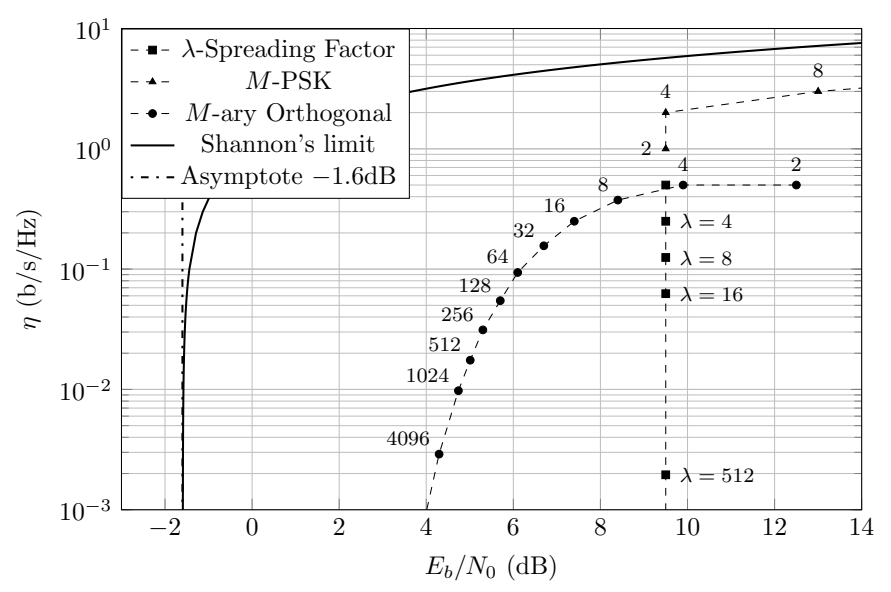

Fig. 1. Spectral efficiency versus $E_{b} / N_{0}$, for different modulations and for $\mathrm{BER}=10^{-5}$.

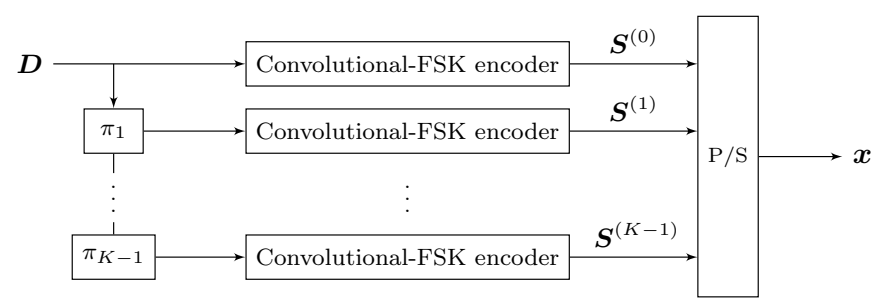

Fig. 2. The Turbo-FSK transmitter with $K$ stages.

The Discrete Fourier Transform (DFT) matrix of size $2^{r} \times 2^{r}$ is denoted $\boldsymbol{F}_{r} . j$ is the complex number, $\bar{z}$ the complex conjugate of $z$ and $\bar{Z}$ is the Hermitian transpose of the matrix $\boldsymbol{Z}$. $\operatorname{Re}(z)$ (resp. $\operatorname{Im}(z)$ ) is the real part (resp. imaginary part) of complex number $z$.

\section{A. Transmitter and Channel}

We denote $D$ the information bit matrix of size $P \times r$, made from $P$ row vectors $\boldsymbol{b}=\left\{b_{n}\right\}_{n \in[0, \ldots r-1]}$. The transmitter is shown in Figure 2. It is composed of $K$ stages, each one having for input an interleaved version of $D$.

The convolutional-FSK encoder is presented in Figure 3. The parity of each row of $\boldsymbol{D}$ is computed, giving the $P \times 1$ parity vector $\boldsymbol{q}^{\prime}$. After passing through the accumulator (representing the row-wise cumulative sum modulus 2 of the elements of $\boldsymbol{q}^{\prime}$ ), the vector $\boldsymbol{q}$ of size $P \times 1$ is obtained. The concatenation of $\boldsymbol{D}$ and $\boldsymbol{q}$ is a $P \times(r+1)$ binary matrix. Each row is an information word, and there are $2^{r+1}$ possible words.

To perform FSK signaling, we map each information word to a FSK symbol taken from the alphabet $\boldsymbol{A}$ (which contains $2^{r+1}$ elements). FSK symbols will be denoted as FSK codewords, each of them being composed of a fixed number of chips (or complex values). $\boldsymbol{A}$ can be constructed using the Hermitian transpose DFT matrix (i.e inverse DFT), $\boldsymbol{A}=\overline{\boldsymbol{F}}_{r+1}$

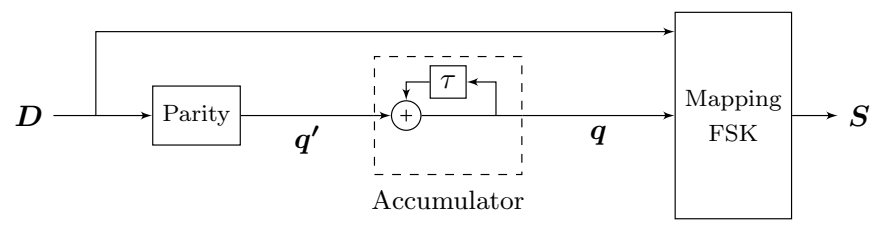

Fig. 3. The convolutional-FSK encoder 


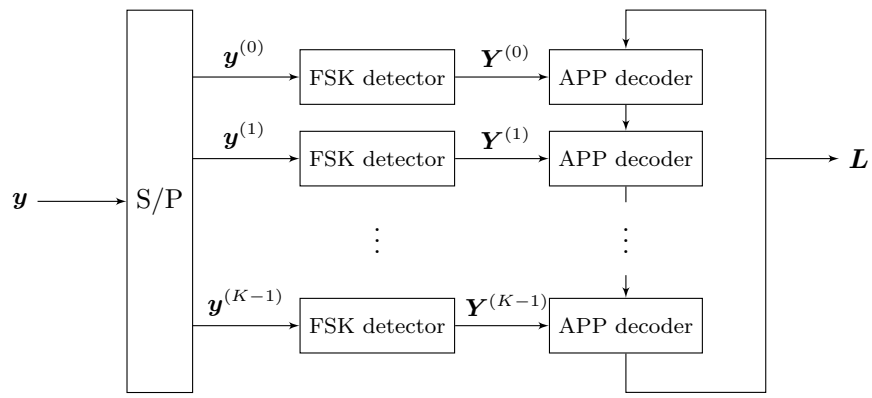

Fig. 4. The Turbo-FSK receiver with $K$ stages.

( $2^{r+1}$ orthogonal codewords composed of $2^{r+1}$ chips), or the concatenation $\boldsymbol{A}=\left[\overline{\boldsymbol{F}}_{r} ; \overline{\boldsymbol{F}}_{r} e^{j \pi}\right]$, a $2^{r+1} \times 2^{r}$ matrix (thus $2^{r+1}$ bi-orthogonal codewords composed of $2^{r}$ chips). The use of bi-orthogonality allows to save one dimension, and will be referred to as the bi-orthogonal case.

The single memory of the accumulator takes part in the mapping of two consecutive FSK codewords. This share of information between codewords will allow the use of the Bahl, Cocke, Jelinek and Raviv (BCJR) algorithm [17] at the decoder side, as introduced in [10].

Each stage output consists of $P$ FSK codewords, that can be multiplexed in quadrature by summing the first $P / 2$ codewords with the last $P / 2$ codewords multiplied by $j$ (assuming $P$ is a multiple of 2). We denote $\alpha P$ the number of output FSK codewords, with $\alpha=1 / 2$ if codewords are sent in quadrature, and $\alpha=1$ otherwise. The output of the $k$-th stage is denoted $\boldsymbol{S}^{(k)}, k \in[0, \ldots K-1]$, and is a $\alpha P \times \mu 2^{r+1}$ matrix, where $\mu=1$ for the orthogonal case, $\mu=1 / 2$ for the bi-orthogonal case. The output $\boldsymbol{x}$ of the Parallel-to-Serial (P/S) block is composed of $\alpha K P$ codewords, or $\alpha \mu K P 2^{r+1}$ chips. It contains the succession of all FSK codewords from each stage. During one codeword period, only one FSK waveform (i.e one pure frequency) is sent if $\alpha=1$, and two FSK waveforms are added in quadrature if $\alpha=1 / 2$.

The spectral efficiency of the Turbo-FSK is

$$
\eta=\frac{r}{\alpha \mu K 2^{r+1}} \text {. }
$$
by

We consider an AWGN channel, which output $\boldsymbol{y}$ is defined

$$
\boldsymbol{y}=\boldsymbol{x}+\boldsymbol{n},
$$

where $\boldsymbol{n}$ is a zero-mean circular complex noise with variance $\sigma^{2}$. All vectors are composed of $\alpha \mu K P 2^{r+1}$ chips.

\section{B. Receiver}

The turbo FSK receiver is depicted Figure 4. After passing through the AWGN channel, $\boldsymbol{y}$ is decomposed, by the Serialto-Parallel (S/P) block, into $K$ vectors $y^{(k)}$, containing the $\alpha P$ codewords of each stage. Each vector is then sent to a FSK detector, that performs the product of the received noisy codewords with $\overline{\boldsymbol{A}}$, i.e the DFT of the vector $\boldsymbol{y}^{(k)}$ (that can be done using FFT algorithm). The outputs $\boldsymbol{Y}^{(k)}$ of the detectors will be used as channel observations in the $a$ posteriori probability (APP) computations, detailed in Section IV and symbolized by the APP decoder blocks. The turbo principle is to iteratively share information among all the decoders. After appropriate interleaving, one decoder will use the information from all the other decoders and the channel observations, to create an estimation of the information bits with more or less likelihood. The $P \times r$ matrix $\boldsymbol{L}$ contains the $\log$ ratio $(\mathrm{LR})$ of the information bits, defined by

$$
L(b)=\log \frac{p(b=1)}{p(b=0)},
$$

where $p(b=0)($ resp. $p(b=1))$ is the probability of event $b=$ 0 (resp. $b=1$ ). The sign of $L(b)$ is the hard bit decision while the magnitude gives the reliability. $\boldsymbol{L}$ is initialized to all-zero and propagated through all decoders. Each one of them uses $\boldsymbol{L}$ as a priori information and updates the matrix with its APP computation. At the end of each iteration, a hard decision can be made from $\boldsymbol{L}$, as it represents all the information acquired.

\section{TURBO-FSK SOFT DECODING}

The principle of soft decoding is to compute the APP knowing an a priori information and the channel observation. For one stage $k$ and case $\alpha=1$, we consider one of the received noisy FSK codeword $\boldsymbol{y}^{p}, p \in[0, \ldots P-1]$ (such as $\left.\boldsymbol{y}^{(k)}=\left[\boldsymbol{y}^{0}, \boldsymbol{y}^{1}, \ldots \boldsymbol{y}^{P-1}\right]\right) . \boldsymbol{d}=\left\{d_{n}\right\}_{n \in[0, \ldots r-1]}$ is the decoded information word. $\boldsymbol{c}^{i}$ is a codeword from the FSK alphabet $\boldsymbol{A}$, and $\boldsymbol{b}^{i}=\left\{b_{n}^{i}\right\}_{n \in[0, \ldots r-1]}$ its associated information word $\left(i \in\left[0, \ldots 2^{r+1}-1\right]\right)$. The APP of a codeword is the probability of having the codeword knowing the observation, and

$$
p\left(\boldsymbol{c}^{i} \mid \boldsymbol{y}^{p}\right)=\frac{p\left(\boldsymbol{y}^{p} \mid \boldsymbol{c}^{i}\right) p\left(\boldsymbol{c}^{i}\right)}{p\left(\boldsymbol{y}^{p}\right)},
$$

by applying Bayes' law. $p\left(\boldsymbol{c}^{i}\right)$ is the a priori probability of having the codeword $c^{i}$, or that the decoded information word $\boldsymbol{d}$ is $\boldsymbol{b}^{i}$. Considering that $\boldsymbol{c}^{i}$ and $\boldsymbol{y}^{p}$ are composed of $\mu 2^{r+1}$ chips, the APP becomes :

$$
p\left(\boldsymbol{c}^{i} \mid \boldsymbol{y}^{p}\right)=\frac{1}{p\left(\boldsymbol{y}^{p}\right)} \prod_{m=0}^{M-1} p\left(y_{m}^{p} \mid c_{m}^{i}\right) \prod_{n=0}^{r-1} p\left(d_{n}=b_{n}^{i}\right),
$$

with $M=\mu 2^{r+1}$. The term $p\left(y_{m}^{p} \mid c_{m}^{i}\right)$ value is, in the complex AWGN case,

$$
p\left(y_{m}^{p} \mid c_{m}^{i}\right)=\frac{1}{2 \pi \sigma^{2}} \exp \left\{-\frac{1}{2 \sigma^{2}}\left\|y_{m}^{p}-c_{m}^{i}\right\|^{2}\right\} .
$$

$y_{m}^{p}$ and $c_{m}^{i}$ being complex numbers, the first product in (8) becomes

$$
\begin{aligned}
\prod_{m=0}^{M-1} p\left(y_{m}^{p} \mid c_{m}^{i}\right) & =B \exp \left\{\frac{1}{\sigma^{2}} \sum_{m=0}^{M-1} \operatorname{Re}\left(y_{m}^{p} \cdot \overline{c_{m}^{i}}\right)\right\} \\
& =B \exp \left\{\frac{1}{\sigma^{2}} \operatorname{Re}\left(Y_{i}^{p}\right)\right\}
\end{aligned}
$$

where $Y_{i}^{p}$ is the $i$-th component of the DFT ${ }^{1}$ of $\boldsymbol{y}^{p}$, and $B$ a constant that will be canceled out in further computations. Using (6) and $p\left(d_{n}=1\right)=1-p\left(d_{n}=0\right)$,

$$
p\left(d_{n}=b_{n}^{i}\right)=C \exp \left\{L\left(d_{n}\right)\left(1-2 b_{n}^{i}\right) / 2\right\}
$$

with $C$ a constant that will be later canceled out. Finally, using (10) and (11), (8) can be expressed as

$$
p\left(\boldsymbol{c}^{\boldsymbol{i}} \mid \boldsymbol{y}^{p}\right)=D \exp \left\{\frac{1}{\sigma^{2}} \operatorname{Re}\left(Y_{i}^{p}\right)+\sum_{n=0}^{r-1} \frac{1-2 b_{n}^{i}}{2} L\left(d_{n}\right)\right\},
$$

${ }^{1}$ For the case $\alpha=1 / 2\left(\right.$ i.e $\left.\boldsymbol{y}^{(k)}=\left[\boldsymbol{y}^{0}+j \boldsymbol{y}^{1}, \ldots, \boldsymbol{y}^{P-2}+j \boldsymbol{y}^{P-1}\right]\right)$, the
real part in (10) would be replaced by the imaginary part for odd values of $p$. 
with $D$ a constant eliminated in further computations. One can notice this expression conveniently uses the result of the DFT of received codeword $\boldsymbol{y}^{p}$ (the observation part, only computed once), and a particular combination of the soft bits $L\left(d_{n}\right)$ (the a priori part, that will be updated at each iteration). By feeding the decoder with the soft bits matrix $\boldsymbol{L}$ and the result of the DFT, the APP of all codewords of the alphabet can be computed using (12).

As previously mentioned, the use of the accumulator and then orthogonal modulation can be seen as sharing information between two consecutive symbols, and codewords probabilities can be updated using the BCJR algorithm. Updated probabilities are denoted $P\left(\boldsymbol{c}^{\boldsymbol{i}} \mid \boldsymbol{y}^{p}\right)$.

Soft bits can be computed [18] with

$$
L\left(d_{n} \mid \boldsymbol{y}^{p}\right)=\log \sum_{i, b_{n}^{i}=1}^{2^{r+1}} P\left(\boldsymbol{c}^{i} \mid \boldsymbol{y}^{p}\right)-\log \sum_{i, b_{n}^{i}=0}^{2^{r+1}} P\left(\boldsymbol{c}^{i} \mid \boldsymbol{y}^{p}\right),
$$

where the indexes of the sums mean probabilities are summed only if $\boldsymbol{c}^{i}$ encodes an information word for which $b_{n}^{i}=1$ (left sum) or 0 (right sum). The factor $D$ in (12) is suppressed at this step.

Complexity of the hereby presented Maximum- $A$ Posteriori (MAP) decoding process can be reduced by using the well-known max-log approximation

$$
\log \left(\sum_{i} e^{x_{i}}\right) \simeq \max _{i}\left(x_{i}\right)
$$

This simplification is done at the expense of some performance loss.

For each decoder, computations can be summarized in three steps:

- Computation of the APP of the $P$ codewords (with (12) $\forall p \in[0, \ldots P-1])$,

- Update the symbols APP using the BCJR algorithm,

- Compute the APP of the information bits by summing the appropriate probabilities of codewords with (13).

\section{Simulation Results}

To illustrate the performance of the Turbo-FSK scheme, simulations were performed for the AWGN channel, with coherent reception and random interleavers. Because the processing gain after 10 iterations is small, our simulations are restricted to this number of iterations.

Figure 5 shows the BER for the case $r=4, K=4$, $P=256$. The transmitted FSK symbols are chosen in an alphabet of size $2^{r+1}=32$. A block of $P \times r=1024$ information bits is considered. We consider here the orthogonal case where symbols are not sent in quadrature $(\alpha=\mu=1$ in (4)). The spectral efficiency is $\eta=1 / 32=0.0312$. The results for both MAP and max-log-MAP algorithms are given. The Block-Error-Rate (BLER) for max-log decoding is also depicted. The difference between exact computation and max$\log$ approximation is roughly equal to $0.5 \mathrm{~dB}$. This performance loss is acceptable considering the reduction of complexity of the decoders, and it should be mentioned that the gap could be reduced by applying a weighting function on the exchanged a priori informations. The spectral efficiency of an orthogonal modulation of same size of alphabet would be $\eta_{1}=(r+1) / 2^{r+1}$; by using the spreading factor scheme to repeat 5 times this orthogonal modulation, the spectral

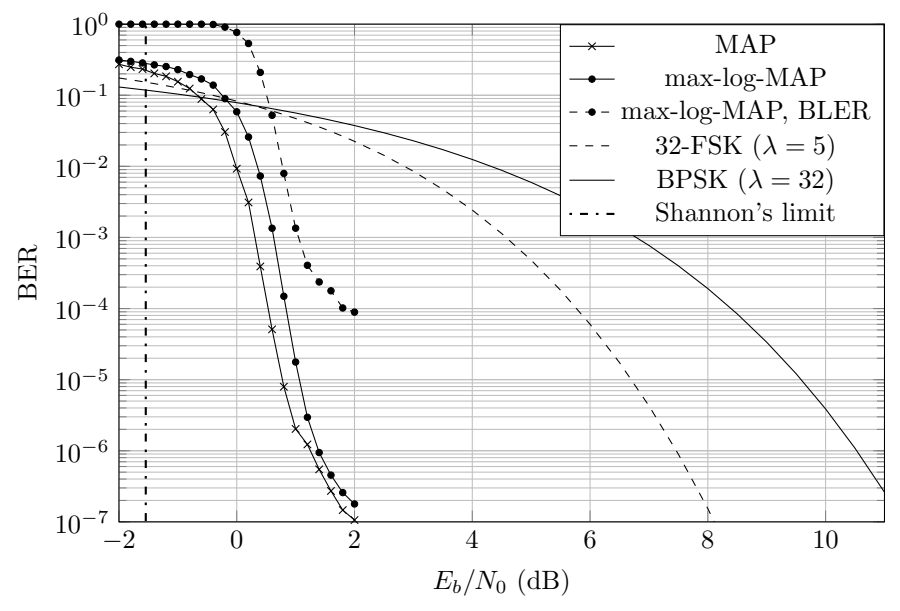

Fig. 5. Turbo-FSK BER performances in $E_{b} / N_{0}$ for the case $r=4, P=$ $256, K=4, \alpha=\mu=1, \eta=0.0312$, after 10 iterations. Shannon's limit is equal to $-1.545 \mathrm{~dB}$ for this spectral efficiency.

efficiency (now equals to $\eta_{1} / 5$ ) is equivalent to the one of the Turbo-FSK with our parameters. The performance gain over repeated 32-FSK is $6 \mathrm{~dB}$ at $\mathrm{BER}=10^{-5}$. Considering now $\lambda$ the spreading factor as introduced in Section II, a spectral efficiency of $1 / 32$ is equivalent to a $\operatorname{BPSK}\left(\eta_{1}=1\right)$ with a spreading factor of $\lambda=32$. At BER $=10^{-5}$, Turbo-FSK shows a $8.9 \mathrm{~dB}$ gain over repeated BPSK. Shannon's limit for the considered spectral efficiency is also represented, showing that with these parameters, Turbo-FSK is only $2.3 \mathrm{~dB}$ away from the capacity limit. At a $E_{b} / N_{0}$ of $1.6 \mathrm{~dB}$, the error floor is reached: BER decreases slower than in the previous waterfall region. The error floor can be shown to be directly related to the values of $K$ and the block size. A BLER of $10^{-3}$ is achieved for an $E_{b} / N_{0}$ of $1.05 \mathrm{~dB}$.

Figure 6 depicts the BER for another case of the TurboFSK, versus the SNR. The considered case is $r=3, K=3$. Multiple values of $P$ are represented, leading to several sizes of block of information bits, from 1023 to 16383 (denoted $N=P \times r)$. The bi-orthogonal case is considered $(\mu=1 / 2$, $\alpha=1$ ). Spectral efficiency is $\eta=0.125=1 / 8$, and is equivalent to an 8 -FSK modulation with spreading factor $\lambda=3$, or BPSK modulation with $\lambda=8$. For a BER of $10^{-5}$, Turbo-FSK with $N=1023$ shows $6.6 \mathrm{~dB}$ of gain versus repeated 8 -FSK and $7.8 \mathrm{~dB}$ gain over repeated BPSK. This $\mathrm{BER}$ is reached for a $E_{b} / N_{0}$ of $1.8 \mathrm{~dB}$ (case $N=1023$ ), and can be compared to the BICM-ID scheme with a similar spectral efficiency presented in [15] (using $N=65536$ ). Our scheme shows an improvement of $1 \mathrm{~dB}$ over BICM-ID, while using a much smaller block size. This BER is achieved at SNR of $-7.24 \mathrm{~dB}$, showing the system is able to work at low levels of SNR. The figure clearly shows the influence of the number of interleaved bits $N$ : increasing this value significantly improves the error floor region. However small block sizes are more suitable to the LPWA context.

Figure 1 has been completed with the results achieved by Turbo-FSK in Figure 7. It shows the evolution of the spectral efficiency depending on the parameters $r$ and $K$, versus $E_{b} / N_{0}$, for a BER of $10^{-5}$. Increasing $K$ from 2 to 3 induces a significant improvement in $E_{b} / N_{0}$, and the gap to the capacity limit is reduced, while spectral efficiency decreases. For $K>4$, improvement in $E_{b} / N_{0}$ is small, and these cases have not been represented. The value of $r$ has a similar effect: 


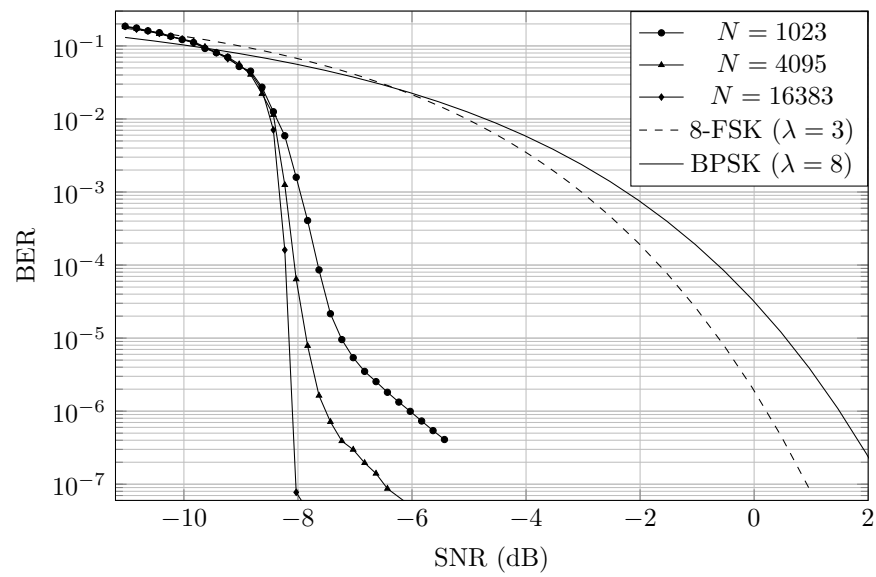

Fig. 6. Turbo-FSK BER performances in SNR for the case $r=3, K=3$, $\alpha=1, \mu=1 / 2, \eta=0.125$, after 10 iterations, for different values of $N=P \times r$. The MAP algorithm was used.

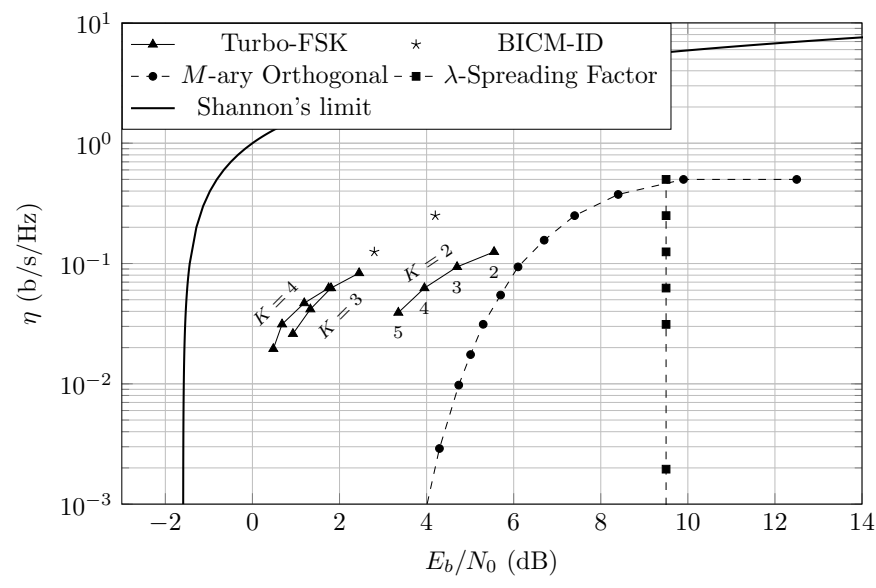

Fig. 7. Spectral efficiency versus $E_{b} / N_{0}$, for BER $=10^{-5}$. Turbo FSK parameters are $\alpha=\mu=1$ and $N=P \times r \simeq 1024$ (as $r$ and $P$ need to be integers). $r$ values are labeled for $K=2$, and identically distributed for other values of $K$. The MAP algorithm was used.

increasing the alphabet allows better performance, while the spectral efficiency is decreased. Setting $r$ to higher values would get the system closer to Shannon's limit, however this may not be sensible in the LPWA context. Moreover, using larger values of $N$ would improve the performance for every case. BICM-ID from [15] is shown on the graph. Results are obtained using a 64-state convolutional code, involving a high decoding complexity due to the Soft-Output Viterbi Algorithm combined with the turbo processing of BICM-ID. Again, the block size considered is $2^{16}$ and is then large compared to the size used in our simulations. For a reduced complexity and smaller block size, our scheme offers better performance.

\section{CONCLUSION}

In the context of M2M communications, terminals are expected to be low power, low cost and to work at very low levels of sensitivity. Used as uplink communication in LPWA network, the newly proposed Turbo-FSK scheme is adapted to these requirements. Simulation results show a significant performance gain that increases with the alphabet size, the number of stages and the information block size. The interleaving strategy of turbo coding is as costly as the repetition scheme, but performance is significantly enhanced thanks to a more complex receiver. The choice of FSK allows the use of basic components to emit the signal (such as a voltage controlled oscillator), and the constant envelope property enable to release some constraints on the power amplifier, because of the low peak-to-average-power ratio of the modulated signal. One way to interpret the observed gains in $E_{b} / N_{0}$ is the reduction of transmitted power at the connected object, while achieving the same level of performance. The system works at low levels of SNR (hence low levels of sensitivity) even when relatively small information block sizes are considered, while the flexibility of available configurations (values of $r, K$ and $P$ ) makes it versatile for various use cases.

\section{REFERENCES}

[1] T. Rebbeck, M. Mackenzie, and N. Afonso, "Low-powered wireless solutions have the potential to increase the M2M market by over 3 billion connections," Analysys Mason, Sept 2014.

[2] A. Ghosh, R. Ratasuk, and N. Mangalvedhe, "Low Power Wide Area Machine-to-Machine Communications using LTE," Nokia presentation, Globecom 14, Dec 2014.

[3] “SigFox website," http://www.sigfox.com/, accessed: May 4, 2015.

[4] "LoRa from Semtech," http://www.semtech.com/wireless-rf/lora.html, accessed: May 4, 2015.

[5] R. Young and D. Zhang, "Introduction to "Clean-Slate" Cellular IoT radio access solution," Huawei presentation.

[6] J. Proakis, Digital Communications 3rd Edition, ser. Communications and signal processing. McGraw-Hill, 1995.

[7] J. Costello, D.J. and J. Forney, G.D., "Channel coding: The road to channel capacity," Proceedings of the IEEE, vol. 95, no. 6, pp. 11501177, June 2007.

[8] C. Berrou, A. Glavieux, and P. Thitimajshima, "Near Shannon limit error-correcting coding and decoding: Turbo-codes. 1," in IEEE International Conference on Communications (ICC). Geneva., vol. 2, May 1993, pp. 1064-1070.

[9] L. Ping and S. Chan, "Iterative decoding of concatenated Hadamard codes," in IEEE International Conference on Communications (ICC)., vol. 1, Jun 1998, pp. 136-140 vol.1.

[10] L. Ping, W. Leung, and K. Y. Wu, "Low-rate turbo-Hadamard codes," IEEE Transactions on Information Theory, vol. 49, no. 12, pp. 32133224, Dec 2003.

[11] Y.-J. Wu and L. Ping, "On the limiting performance of turbo-Hadamard codes," Communications Letters, IEEE, vol. 8, no. 7, pp. 449-451, July 2004.

[12] N. Shimanuki, B. M. Kurkoski, K. Yamaguchi, and K. Kobayashi, "Improvements and extensions of low-rate Turbo-Hadamard codes," International Symposium on Information Theory and its Applications, ISITA2006, Nov 2006.

[13] K. Kikuchi and M. Osaki, "Highly-sensitive coherent optical detection of M-ary frequency-shift keying signal," Optics Express, vol. 19, no. 26, pp. B32-B39, 2011.

[14] P. Liang and W. Stark, "Algorithm for joint decoding of turbo codes and M-ary orthogonal modulation," in IEEE International Symposium on Information Theory., 2000, p. 191.

[15] J. Panaro, "Simple iterative decoding for bit-interleaved coded orthogonal modulation," in Joint IST Workshop on Mobile Future. SympoTIC '06., June 2006, pp. 16-19.

[16] C. Shannon, "A mathematical theory of communication," The Bell System Technical Journal, vol. 27, no. 3, pp. 379-423, July 1948.

[17] L. Bahl, J. Cocke, F. Jelinek, and J. Raviv, "Optimal decoding of linear codes for minimizing symbol error rate (corresp.)," IEEE Transactions on Information Theory, vol. 20, no. 2, pp. 284-287, Mar 1974.

[18] J. Hagenauer, E. Offer, and L. Papke, "Iterative decoding of binary block and convolutional codes," IEEE Transactions on Information Theory, vol. 42, no. 2, pp. 429-445, Mar 1996. 